\title{
Understanding User Requirements for the Design of a Semantic Digital Library Interface
}

\author{
Cristina Emilia Costa ${ }^{1}$ and Marco Ronchetti ${ }^{2}$ \\ ${ }^{1}$ CREATE-NET, Trento, Italy \\ cristina.costa@create-net.org \\ ${ }^{2}$ DISI, University of Trento, Trento, Italy \\ marco.ronchetti@unitn.it
}

\begin{abstract}
This paper discusses the role of interaction and user experience in the design of an interface of search for digital libraries. We present our preliminary work in the design of an advanced interaction interface for "Publicamente", a web based semantic multimedia digital library that allows the archiving, management and access to a collection of heterogeneous documents types. In this paper we explore the user requirements, and present the design guidelines for the interface of the system. Users of "Publicamente" are be both expert users and casual users and the interface need to being able to accommodate different search styles, make semantic technologies transparent to casual users, and leverage on the semantic layer in the presentation of the retrieved information and its context.
\end{abstract}

Keywords: HCI, search user interface, digital libraries.

\section{Introduction}

A digital library is an information system designed to collect, organize, retrieve and access digital documents, integrating them with information coming from metadata from different sources. The search user interface plays an important role in it, since it supports the multiple functions needed during information search. First of all it has to support the user, or searcher, in formulating the more appropriate query for his needs or in browsing through the content available. Then, it should help him in the understanding and interpretation of the search results, subsequently aiding him in the selection of the content more appropriate to his information needs. Finally, it should keep track of the recent search activities, making them accessible during the search session in order to allow the user to improve and refine his research.

In modern digital libraries, text based documents are placed side by side to multimedia content, and the information is distributed over multiple and mixed formats. Besides, the role of multimedia has gradually shifted from being mostly entertaining to informative or documentary. These factors influence both the search and fruition of data, making the design of the user interface more challenging and complex.

Recently research has focused in making multimedia content fully searchable and easy to access as traditional text documents, using various techniques able to enrich 
the interpretation of the content, such as text to speech converters, speaker recognition, and automatic evaluation of the visual content.

In some types of digital libraries, such those containing content from conferences, lectures, formal meetings or public administration activity documentation, contents with different formats are often related to each other. These relationships are part of a context that plays an important role in the comprehension of the documents themselves, and therefore they should be preserved, and correctly presented in the visualization interface.

The introduction of semantic technologies empowers the search, making it more robust, improving results presentation that can be supported by significant contextual information. Indeed, being able to work with semantically enriched documents, especially if extended to multimedia, allows emerging significant relationships between documents and contextual information.

\section{The "Publicamente" Platform}

"Publicamente" is a web based semantic multimedia digital library that allows the archiving, management and access to a collection of heterogeneous documents types. "Publicamente" is able to manage a wide variety of content types: documents may be video, audio (podcasts), images and text based documents (such as PDF, HTML, PowerPoint, Word, OpenOffice, and Excel document formats).

The platform was created to support digital libraries where multimedia is predominant and where the content is connected to each other by temporal and location constraints, such as in documentation from conferences, lectures, meetings, etc.

"Publicamente" groups related documents, and their metadata information, into "event" entities, and uses additional metadata to markup documents with time, date and location. These entities may be assimilated to a collection of temporal related content, where various heterogeneous types of documents that are related to each other are grouped together forming a complex entity, where information is delivered as a whole over multiple heterogeneous channels. The semantic layer is able to link the groups (or collections) of documents with others in the library and assists the users in the retrieval of the content relevant with respect to their information needs. This structure is then used to search and access data and to display the content taking into account user preferences.

Presenting the entity as a whole gives the advantage to emphasize the context in which the content has been created.

Since video is predominant in this type of digital library, the platform fully supports features designed for dealing with video based content. Video can be searched through the transcripts of the audio (either already available in form of subtitles or automatically generated thanks to a speech to text engine), or through other types of synchronized content (e.g. slides). Therefore, besides text documents, also videos can be semantically enriched with automatic processes. Search result contains the exact time stamp in which the information was retrieved, allowing the user to quickly check the results. 


\section{Use Cases}

"Publicamente" is a platform that gives access to the general public to documentation on specific topics. The search system interface is the main point of access for the user to the digital library, and it has an important influence in the user experience (UX) and on the accessibility of the content. We consider as potential users of the system both expert users and casual users: therefore the interface needs to be able to accommodate different search styles, make semantic technologies transparent to casual users, and leverage on the semantic layer in the presentation of the retrieved information and its context.

The interface should be intuitive and graphically appealing in order to satisfy the needs of first time or casual users, supporting both query search and explorative search. These users may expose a more exploratory form of search, for example for learning about a topic, discover events, and understand complex topics as a part of a course. At the same it should support also expert groups of users that need to search specific information or documents in the library. The objectives and motivations of these two user groups, as well as the type of information search, may therefore be quite different. Due to the specific type of content, there is need for an interaction interface that does not just return results but is also able to provide an effective user experience, letting users to see and explore the richer context connected to the documents.

Since we are working with a heterogeneous collection of information, depending on the nature of the content (audio, video, text) it requires to be read, seen or listen. The interface should not only give the user the control over his findings, but also provide easy and practical tools for interacting with the content and checking their interest on it.

For defining the principles of the design of the user interface we defined tree real world use cases that generalize tree implementations of the system.

\subsection{Event Document Repository}

Big recurrent events such as Festivals and Expos produce in time a large library that archives various types of connected documentation and represents a history memory of the event. "Publicamente" is used to create a portal that collects a great variety of contents over a single topic (e.g. Economy) provided in various formats such as video of keynotes, audio of interviews, slides, photographs, biographies of speakers. The portal allows retrieving documents related to a specific moment of the event, such as a keynote (and related documents) as well allow exploratory search, but the more interesting feature its capability in making connections between past and new information, thus allowing emerging the evolution of a specific topic in time, and its key players.

\subsection{Academic Environment}

In academic environment "Publicamente" can be used to create a portal that collects courses and related lecture materials. Lecture content is by its nature mixed media, 
containing videos, presentations and reading material. These can be enriched with information from the academic library and journals. The semantic organization of the information allow to make cross-links between materials of various courses (for example linking material from 101 courses to more advanced ones), helping students to connect information acquired in different moments of their academic path.

\subsection{Public Administration}

Transparency in the political life of public administration is becoming a more and more central issue for local communities. For increasing the access of citizen to the information discussed during debates, sometimes these are video recorded and made available to the public. Unfortunately being able to access to recorded information without other forms of support does not help the citizen in find in an efficient way the information he needs."Publicamente" allows creating an archive of the information indexing the video information and connecting it to textual documentation, such as minutes and resolutions, and to profiles of the politicians involved.

\section{Advanced Interaction Interface Design}

In "Publicamente" it is fundamental that the visualization and interaction strategy adopted in the interface is able to reflect the information needs that drive the users' search in each use case. The main common element that emerges is the importance of context in which the document is created and subsequent referred to. The user interface should present to the user the semantic enrichments and the connections with other documents in a form that he can use it.

Publicamente requires an advanced interaction interface, able to efficiently present to the user a collection of heterogeneous documents types and is able to manage a wide variety of content types: documents may be video, audio (podcasts), images and text based documents (such as PDF, HTML PowerPoint, Word, OpenOffice, and Excel document formats), supporting the range of use cases and finding an interface that best if fits into more than one category.

The use of interactive and graphical information has the goal of amplifying and reinforcing human cognition, thus enabling the viewer to gain knowledge about the internal relationships between documents and information, and making it emerge the structure of the library organization. The interface supports human interaction for exploration and understanding using graphical and layout elements for visualizing context elements (being it metadata such temporal, meaning, author information, relationships or semantic enrichment) and mapping them in the screen, taking advantage of psychological principles, such as selective proximity of the search results, alignment of the elements, and chosen shared visual properties such as color or size.

Diversity of users search styles is supported by exploiting the interaction properties of the interface: for example selective hiding of data contributes keeping the displayed information clean and simple, while additional details remains available to users that require deeper information; giving more space to the information related to 
key documents help to maintain the focus of the search. Interaction can be designed to enable users to make discoveries, getting understanding and take decisions and adapting the layout as the user refines the search.

In the design of the search user interface (SUI), we take into consideration the four types of features [3]:

- Input Features: search box based (natural language query), facets, clusters categories, ontology, context

- Control Features: Sorting, filters, grouping, suggestions

- Informational Features: previews, usable information, social information

- Personalisable Features: current search, persistent

Each of these features contributes to improve the user search experience: the ideal SUI should include elements that combine as more of these features as possible. For understanding from the usability point of view what makes search easy or difficult, we analyzed the interface so that it could support each phase of the four-phase search framework [2] mapped in the range of the envisioned use cases:

- Formulation, represents what happens before the search. It is connected with the objectives of the user and with the motivation that brings him to the portal.

- Action, which type o search he performs.

- Review of results, presentation of the found data

- Refinement, improving the search

The interface should be keep simple ("produce a search interface that is both highly functional, but not cluttered and distracting" [1]), but allow various levels of exploration.

\section{Conclusions}

During the design process of the user interface of the "Publicamente" for different use case, some key characteristics of the platform emerged. In particular two aspects needed $n$ special attention. First the presentation of the search results that involved multimedia content: it is not sufficient to list audio or video titles, but it is necessary to give the user a quick access to them in relevant points, fully exploiting in the presentation the richer semantic information available. Secondly, the organization of documents in logical entities need to be supported and visualized side by side to other less structured connections between documents suggested by the semantic engine. These connections, enriched by metadata information, create the context around the documents during the search, and contribute to the correct interpretation of the found results.

Acknowledgements. This work was partially supported by the Publicamente project, co-funded by the Province of Trento (PAT) under the POR FESR 2007-2013 EU program, bando n.2/2010. 


\section{References}

1. Wilson, M.L., Kules, B., Schraefel, M.C., Shneiderman, B.: From Keyword Search to Exploration: Designing Future Search Interfaces for the Web. Found. Trends Web Sci. 2(1), 1-97 (2010), http: / / dx.doi.org/10.1561/1800000003, doi:10.1561/1800000003

2. Shneiderman, B., Byrd, D., Bruce Croft, W.: Sorting out searching: a user-interface framework for text searches. Commun. ACM 41(4), 95-98 (1998), http: / doi . acm . org / 10.1145/273035.273069, doi:10.1145/273035.273069

3. Wilson, M.L.: Search User Interface Design. In: Marchionini, G. (ed.) Synthesis Lectures on Information Concepts, Retrieval, and Services. Morgan \& Claypool (2011) 